\title{
IRECIST Complete Response
}

National Cancer Institute

\section{Source}

National Cancer Institute. iRECIST Complete Response. NCI Thesaurus. Code C142357.

The disappearance of all signs of cancer following an initial apparent radiographic progression. 